\title{
Simulation of Vehicle's Frontal Crash with Dummy inside
}

\author{
LiuYuanpeng \\ Research Institute of Highway Ministry of Transpor \\ Beijing, 100088. China \\ E-mail: yp.liu@rioh.cn
}

\begin{abstract}
The complete Finite Element Model of vehicle with dummy inside is established. Through analyzing structure deformation and acceleration of the vehicle, the rule of energy absorption and dissipation is obtained, the dummy's respond and the collision capability criteria of the head, chest and thigh are achieved. A comprehensive and credible appraisement about the frontal crash process and crashworthiness is proposed by analyzing the effect of the main energy-absorbing components, the transmitted route of the energy and the safety of the vehicle and occupant injury criterions.
\end{abstract}

Keywords- Frontal crash; Dummy; FEM; Energy absorption; Simulation

\section{INTRODUCTION}

Nowadays, vehicles are designed to have higher speed and lighter weight, traffic accidents and the casualties sharply increase. In order to enhance passivity security of vehicles, the crash passiveness characteristic and protecting passengers of vehicles structure must be studied in design stage [1]. Although the vehicles crash testing is necessary to appraising vehicles and passiveness protection device, test expenditure is too expensive and the test result is not stable for random factor. Vehicles crash simulation including dummy can accurately forecast not only the crash characteristic of vehicles but also response and injury of passengers in crash process. It is also very helpful for the development of the new and helpful in reducing chances of the collision of cars in a shorter term and at a lower cost.

In this paper, the complete finite element models of a passenger car and the occupant restraint system including a 50th percentile Hybrid III dummy and the seatbelt subsystem are set up. According to the CMVDR 294, the frontal crash into a rigid wall of the car at about $48.3 \mathrm{~km} / \mathrm{h}$ is successfully simulated in LS-DYNA and the results including the car's displacement, velocity, the time course of the acceleration, the transmitted interrelation of the energy, the dummy's injury value, achieve comprehensive and credible appraisement with the frontal crash process and crash performance. It provides the theoretical reference for the studies of the crash process and the crashworthiness design of vehicles.

\section{ESTABLISHMENT OF CRASH MODEL}

This paper establishes the numerical modeling of the vehicle's parts in the CATIA, and connected the geometric modeling of the whole vehicle body. Importing the numerical modeling of the vehicle into the preprocessor environment of the HypeMesh, and meshing elements. Basing on the structure of body-in-white, this paper increased the engine, assistant suspension, seat, front and back doors, suspension and the wheel assembly and so on [24]. Simultaneously the parts, whose stiffness is harder, such as the engine, transmission, etc, are considered as rigid body. And adding the established finite element dummy model into the vehicle, adjusting the sitting posture and restriction, completes FEM of the integrated occupant restraint system, as shown in Figure 1.

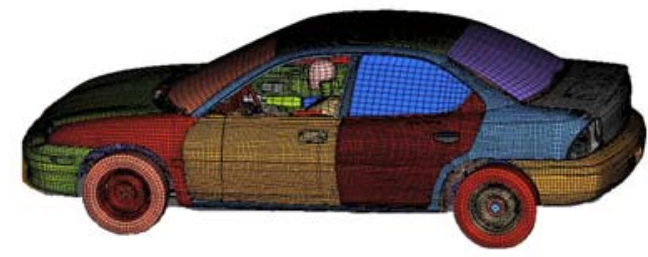

Figure 1. The FE Model of the Car

This paper adopts Belytschko-Tsay shell element to calculate in the course of simulating sheet metal component of vehicle body' structure. Material properties adopt the Multi-linear elastic plasticity Isotropic hardening material [5]. Distribution of meshing of the whole vehicle model: the norm size of finite element meshing of the whole vehicle should be $10 \mathrm{~mm}$ in order to meet the demand of both frontal crash and side crash of finite element model. We adopt the reducing integral element with hourglass controlling, and control the characteristic size of minimum element at about $5 \mathrm{~mm}$. There are 320,000 nodes and 300,000 elements in the whole vehicle finite element model.

\section{THE OCCUPANT RESTRAINT SYSTEM}

\section{A. The Dummy Finite Element Model}

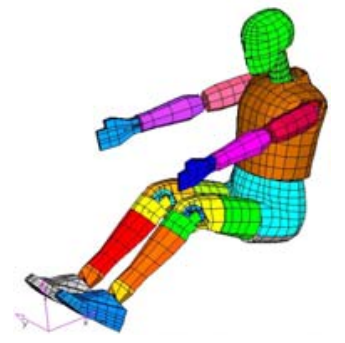

Figure 2. Hybrid III dummy

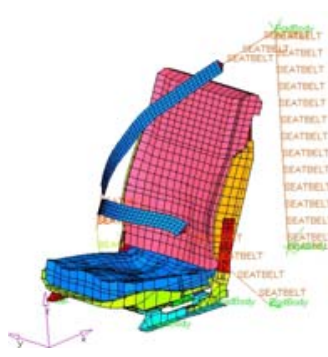

Figure 3. seatbelt model
The dummy finite element model, which was used to emulate, is completely based on the test of 50th percentile 
Hybrid III dummy, as shown in Figure 2. The key parts, such as chest and neck, are mounted with the quality of flexible, so as to judge of the injury after crash [6].

\section{B. The Seatbelt Model}

The dummy is constrained by three-spot style seatbelt, as shown in Figure 3. The seatbelt finite element models include seatbelt, slip ring, pretensioner, retractor and the sensor element that can make pretensioner and retractor working. The seatbelt model at the front of the chest is simulated by the two-dimensional shell element.

\section{The Enactment of Contact Type}

There are contacts between the dummy's chest and the seatbelt, dummy's waist and the waistband, dummy and the front floor of the whole vehicle, the cushion of the seat and the support parts of the seat, the vehicle and the rigid wall, the main parts of body, when the vehicle collided.

The precise definition of the contact type is essential to the solution. And it is difficult to judge the contact direction of the shell element in the crash process. So we should try to introduce the AUTOMATIC contact type. This type is mainly exploited to aim at the direction of the shell element, and can fit many kinds of contact action perfectly [2][4].

\section{SimULATION AND APPRAISEMENT}

\section{A. The Analysis of the Whole Vehicle's Distortion}

From Figure. 4 show the distortion of the whole vehicle structure and the response of dummy at the time $100 \mathrm{~ms}$. The bumper, engine cover and the front longitudinal member have generated large plastic deformation, pinching together with the front component of the front longitudinal member, and the front longitudinal member which have shown large folding deformation takes effects in absorbing the energy. At the same time, the cockpit and the rear of the vehicle haven't shown deformation.

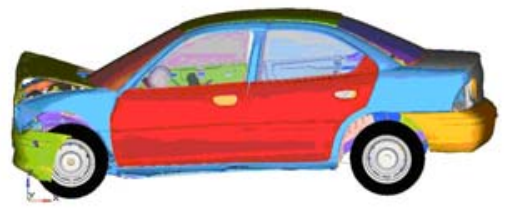

Figure 4. Frontal crash simulation result in $100 \mathrm{~ms}$

The reason of the phenomenon is with the occur of collision, the front of the vehicle have taken great impact, so the impact energy is tremendous, and the momentum changes rapidly in a very short time, it forms impact force in a moment, and the number is very large. The front of the vehicle body with the great impact force causes the excessive stress surpassing the material bend stress greatly, so it can result in greater plastic deformation. At the same time, it can absorb the impact force mostly, so the kinetic energy of the vehicle decreased and the number attenuates obviously, and the stress which effects on the cockpit and the rear of the vehicle body also comes down, haven't resulted in obvious deformation.

\section{B. The Change of the Whole Vehicle's Displacement}

At the vehicle we take the change of displacement of one given point (node 170805) in A-pillar relative to the other point (node 170654) in B-pillar, as the Figure.5 shows. From that we can know the maximum displacement on node 170805 relative to node 170654 in $\mathrm{X}$ direction is about $11.7 \mathrm{~mm}$, this displacement is not enough to pose crisis to the living space of driver, and it show that the crashworthiness of the vehicle is good. Further more, the $11.7 \mathrm{~mm}$ displacement is also small to the door displacement. The door can be opened without any other tools after crash, which can make the driver leave the crashed vehicle conveniently.

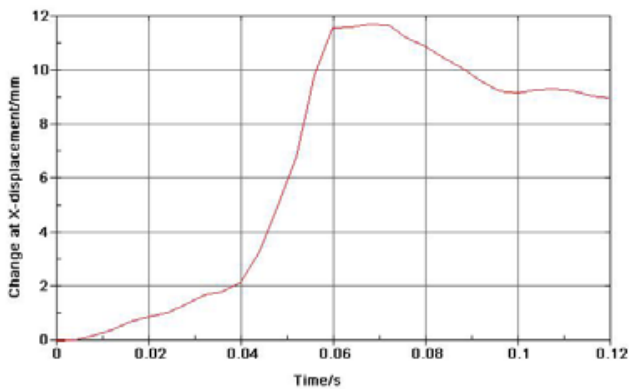

Figure 5. The displacement of node 170805 relative to node 170654

As to the occupant restriction system, we adopt the deformation of firewall relative to floor structure to analyze. The Figure 6 shows the displacement of one given point (134931) at firewall relative to the other point (183419) at floor, the maximum is $43 \mathrm{~mm}$, the structure deformation cause little effect to the thigh and its space.

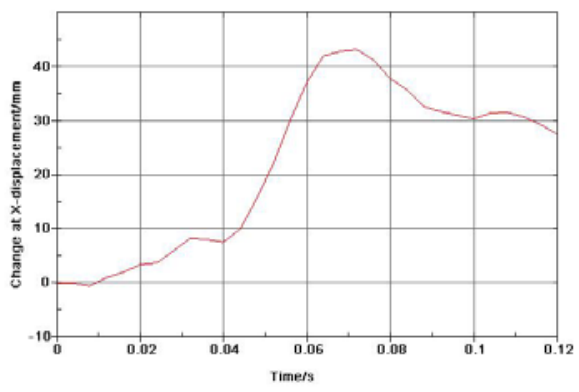

Figure 6. The displacement of Node 134931 relative to node 183419

\section{The Change of the Whole Vehicle's Velocity}

As the beginning speed is $13.4 \mathrm{~m} / \mathrm{s}$, we take the front, middle and back section in all three point(node 384744 , node 171710 and node 166579) to analyze the change of speed of varying vehicle section. As the Figure 7 shows, the speed on node 3847449(a point in bumper) falls down the most quickly, at time $5 \mathrm{~ms}$ it has come down to zero. The speed on node 171710(a point in B-pillar) and node 166579(a point in the rear of the vehicle ) falls down both to zero at about time $64 \mathrm{~ms}$, but it has reversal speed after falling down to zero, 
this reflects the phenomena that the vehicle would generate rebound after crash.

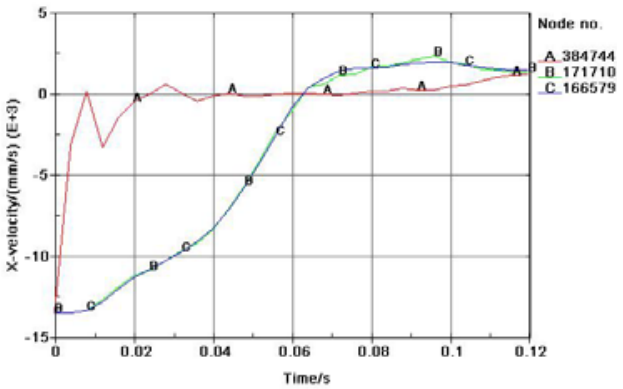

Figure 7. The time courses of vehicle's velocity

\section{The Change of the Whole Vehicle's Acceleration}

Acceleration is one of the most important criteria when evaluating the passive security of the vehicle's structure. Because the excessive acceleration will cause the re-crash between the occupant and the vehicle arouse accident. The analysis of the acceleration of the vehicle can recur to the measure of the acceleration of the B-pillar. The Figure 8 is the curve of the acceleration of the node 113061 which is one given point of B-pillar. The peak of the acceleration is about $67 \mathrm{~g}$.

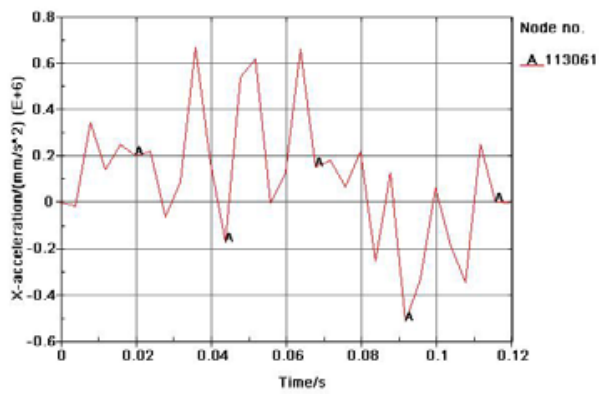

Figure 8 . The time courses of vehicle's acceleration

\section{E. The Analysis of the Energy Result}

The Figure. 9 can reflect the change of the energy during the process of the crash. The maximal kinetic energy of the system is $117 \mathrm{KJ}$. It descends sharply with the process of the crash. At the same time, due to the distortion of the components, which can absorb the energy, the internal energy ascends sharply. Up to the end of the crash, $90 \%$ kinetic energy has been transformed to the internal energy of distorted components. Because of the rebound of the vehicle at the end of crash, the kinetic energy has not descended to zero; it still remains $2 \mathrm{KJ}$ or so.

In the process of crash, the front energy-absorbing components absorb the main internal energy and try to stop passing or only permit a little energy to the occupant-cabin, for keeping the space of the occupant unchanged. The energy-absorbing components vary according to the time of the absorbing.

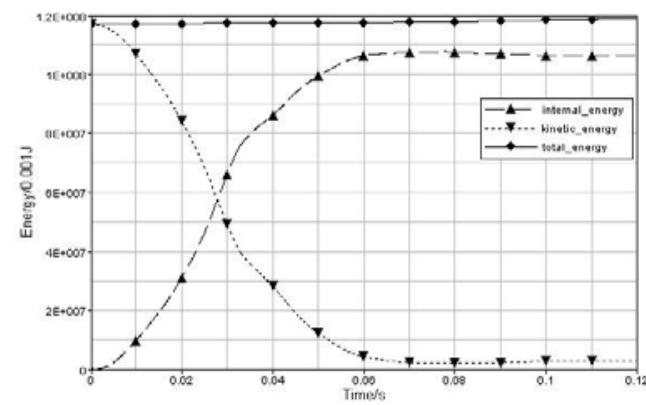

Figure 9. The change of the energy

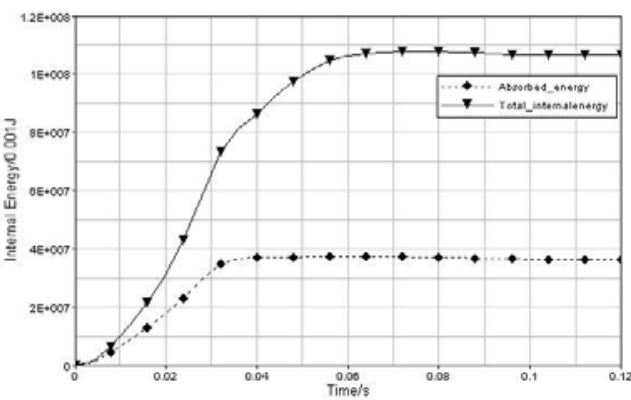

Figure 10. The energy absorption curve

Between 50ms and 60ms, main energy-absorbing components are the extension of longitude member, firewall, side panel, the floor and so on. The energy-absorbing components analyzed above absorb about $70 \%$ of the total internal energy. The other front components of the vehicle such as engine can also absorb part of internal energy. So the front components of the vehicle absorb most of the internal energy[10].

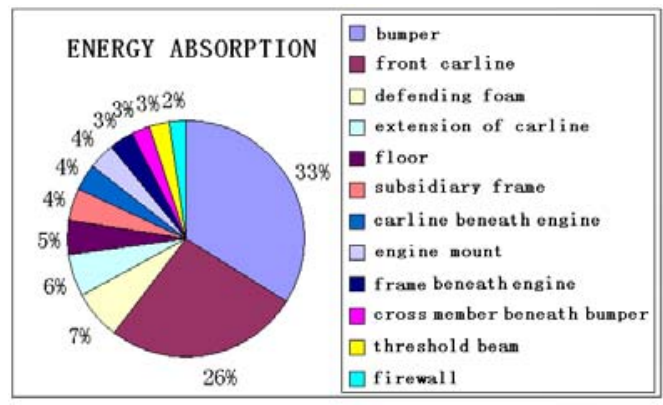

Figure 11. The adsorbed energy ratio in front crash

Figure 11 shows the condition of the main energyabsorbing components in the process of crash, we can see the front bumper absorbs $20 \mathrm{KJ}$ internal energy which occupies about $20 \%$ of the total energy. The effect of energy absorption is good the longitude member absorbs $14.5 \mathrm{KJ}$ internal energy and the effect is not better than the bumper. We can consider changing the energy absorption mode of the front longitude member by adding buffer components between the front bumper and the front longitude member. As one part of the front longitude member assembly welldesigning the crash intension of the buffer can make the new 
front longitude member produce folded failure mode in phase and then cause the succeeding failure of the behind longitude member structure. So it can improve energy absorption of the front longitude member assembly structure in the process of crash [7-9].

\section{F. The Analysis of the Dummy Response}

The response of dummy in the process of crash is shown in Figure 12 At the time of $20 \mathrm{~ms}$ dummy's back begins to leave the backrest and move forward with its neck a little bending; when the time is $40 \mathrm{~ms}$, the vehicle's deformation rises and dummy keeps moving forward, the head has a trend to decline, the arms moves forward and the legs begins to bend as well; when the time is $60 \mathrm{~ms}$, the vehicle's deformation keeps rising, the dummy as a whole slides forward along the seat, the head declines, the arms keep moving forward and the legs bend with a larger range; when the time is $80 \mathrm{~ms}$, the front of the vehicle deforms seriously. The head contact steering wheel, and the dummy begin to crouch; when the time is $100 \mathrm{~ms}$, the body keeps crouching, the head crashes the steering wheel and reaches its lowest point.

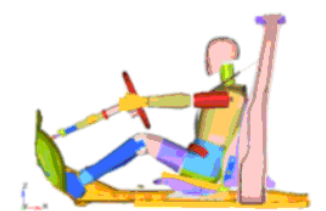

Oms

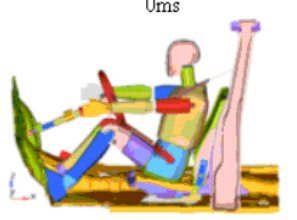

$40 \mathrm{~ms}$

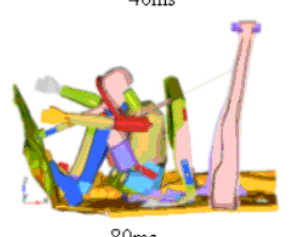

$80 \mathrm{~ms}$

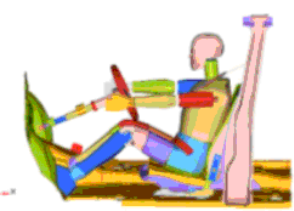

$20 \mathrm{~ms}$

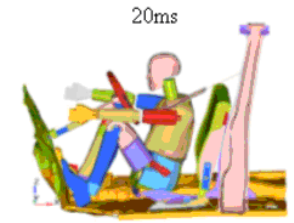

$60 \mathrm{~ms}$

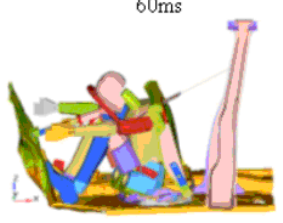

$100 \mathrm{~ms}$
Figure 12. The response of dummy in the process of crash

\section{G. Injury Criterions and Dummy Appraisement}

The criteria adopted to evaluate the security capability are the Injury criterions of dummy which prescribed in the rule of CMVDR294 and the chest acceleration criterions which prescribed in occidental regulation. The compare of simulated value and injury criterions of dummy is shown in the table 1 .

\section{TABLE I. THE COMPARE OF INJURY CRITERIONS}

\begin{tabular}{|c|c|c|}
\hline Injury criterions & Simulative value & Requirement \\
\hline $\mathrm{HIC}$ & 843.5 & $\leq 1000$ \\
\hline $\mathrm{THPC}$ & $34 \mathrm{~mm}$ & $\leq 50 \mathrm{~mm}$ \\
\hline $\mathrm{C}_{3 \mathrm{~ms}}$ & $56 \mathrm{~g}$ & $<60 \mathrm{~g}$ \\
\hline
\end{tabular}

\begin{tabular}{|c|c|c|}
\hline \multirow{2}{*}{ FPC } & $2.15 \mathrm{KN}$ (left) & \multirow{2}{*}{$\leq 10 \mathrm{KN}$} \\
\cline { 2 - 2 } & $1.23 \mathrm{KN}$ (right) & \\
\hline
\end{tabular}

In table 1, the criterion of HIC is worked out by CMVDR 294 and occidental rule based on formula (1).

$$
H I C=\left\{\left[\frac{1}{t_{2}-t_{1}} \int_{t_{1}}^{t_{2}} a(t) d t\right]^{2.5}\left(t_{2}-t_{1}\right)\right\}<1000
$$

where $a(t)$ is the resultant linear acceleration time history (G's) of the center of gravity of the head, and $t_{1}$ and $t_{2}$ the interval between every $36 \mathrm{~ms}$ of the crash process which produced the maximal HIC. $\mathrm{C}_{3 \mathrm{~ms}}$ is the $3 \mathrm{~ms}$ criterion of the chest acceleration.

From the analysis we can know that the deflection values of the dummy's head, chest and thigh meet with the requirement of collision regulation collision well. The design of this kind of car is successful according to the crash security.

\section{CONCLUSION}

1. All the guidelines of crash estimation of the vehicle, which has good passive safety, are quite enough to meet the requirement of crash regulation.

2. The energy-absorbing parts at the front of the car, which have a good effect to absorb the energy, absorb most of the internal energy. The design of the front longitudinal member should be improved because it has less effect for absorbing the energy.

3. The deflection values of the dummy's head, chest and thigh are enough to meet the crash regulations requirement. The simulated testing results indicate that the safety of the vehicle is good when taking place the frontal crash.

\section{REFERENCES}

[1] Moisey B.shkolnikov, "Strain Rates Crashworthiness", 8th Internationl LS-DYNA Users Conference, 2004, pp. 83-95.

[2] Livemore Software Technology Corporation, Ls-Dyna Keyword User's Manual, California: Livemore Software Technology Corporation, 2001, pp.177-184,251-260, 565-572, 669-675.

[3] AjitD.kelkar, Mark J.Schulz, "Simulation of a Car Frontal Offset Impact into a Fixed Deformable Barrier", SAE Paper, 962458, 1996.

[4] H.o. Zhao, "The guide of LS-DYNA dynamical analyze", Beijing, Weapon Industry Press, 2003, pp. 34-35.

[5] Livemore Software Technology Corporation, "LS-DYNA KEYWORD USER'S MANUAL”, April 2003 Version 970. 2003, pp. 66-70.

[6] Jac Wismans, Future of human models for crash analysis, IPC2001E225, 2001

[7] H.Y.Liang, Q.Guan, J.W. Chen, The Analyze of the Rule about the Face-collision Examination in China, the World of Car, 2001, 11: pp. 12-13.

[8] Z.H.Zhong, W.G.Zhang, L.B.Cao, "The Safe Technology of Vehicle Collision”, Beijing, China Machine Press, JULY 2003.

[9] Iulian Lupea, Joel Cormier and Sital Shah, "FEM for Impact Energy Absorption with Safety Plastic", 8th Internationl LS-DYNA Users Conference, 2004, pp. 65-74

[10] G.S.Zhang, W.Wang, Y.Chen, T.X.Zhang. "Simulation and Experiment of Front Crash of Vehicle", Journal of Northeastern University, 2011, pp. 1468-1470. 